\title{
X-ray standing wave and reflectometric characterization of multilayer structures
}

\author{
S. K. Ghose and B. N. Dev* \\ Institute of Physics, Sachivalaya Marg, Bhubaneswar - 751 005, India
}

\begin{abstract}
Microstructural characterization of synthetic periodic multilayers by $\mathrm{x}$-ray standing waves have been presented. It has been shown that the analysis of multilayers by combined x-ray reflectometry (XRR) and x-ray standing wave (XSW) techniques can overcome the deficiencies of the individual techniques in microstructural analysis. While interface roughnesses are more accurately determined by the XRR technique, layer composition is more accurately determined by the XSW technique where an element is directly identified by its characteristic emission. These aspects have been explained with an example of a 20 period $\mathrm{Pt} / \mathrm{C}$ multilayer. The composition of the C-layers due to $\mathrm{Pt}$ dissolution in the C-layers, $\mathrm{Pt}_{x} \mathrm{C}_{1-x}$, has been determined by the XSW technique. In the XSW analysis when the whole amount of Pt present in the C-layers is assumed to be within the broadened interface, it leads to larger interface roughness values, inconsistent with those determined by the XRR technique. Constraining the interface roughness values to those determined by the XRR technique, requires an additional amount of dissolved $\mathrm{Pt}$ in the C-layers to explain the Pt fluorescence yield excited by the standing wave field. This analysis provides the average composition $\mathrm{Pt}_{x} \mathrm{C}_{1-x}$ of the C-layers.

PACS numbers: 07.85.-m, 61.10.Kw, 68.35Dv
\end{abstract}

\section{INTRODUCTION}

Improvements in the thin film deposition techniques in recent years have led to the fabrication of layered synthetic microstructures (LSM) consisting of thin layers of alternating elements or compounds [1,2]. These materials have unique structural [3], magnetic [4] and electronic [5] properties with a wide range of applications. LSM containing alternating layers of high atomic number elements (eg., W, Mo, Pt etc.) and low atomic number elements (eg., C, Si etc.) are being used as x-ray reflectors $[6]$. Indeed, x-ray multilayer optics are now used in many applications including x-ray astronomy, microscopy, spectroscopy, as filters and monochromators for intense sources such as synchrotron radiation and x-ray laser cavities. It is important to correlate the measured properties with structure so that preparation techniques can be optimized to yield high performance materials. $\mathrm{X}$-ray techniques are very useful for the measurement of the microstructural aspects of the multilayered systems. Here we present the application of combined x-ray standing wave and x-ray reflectometry techniques for the microstructural analysis of periodic multilayers.

For a perfect single crystal, according to the dynamical theory of x-ray diffraction [7,8], a standing wave field is generated within the crystal as a result of superposition of the incident and the diffracted waves when x-rays are Bragg-reflected by the crystal. The equi-intensity planes of the standing wave field are parallel to and have the periodicity of the diffracting planes. At an angle of incidence corresponding to the rising edge of the diffraction peak the antinodal planes of the standing wave field lie between the diffracting planes. As the angle of incidence increases the antinodal planes move continuously inward onto the diffracting planes at the falling edge of the diffraction peak. Over the angular region of Bragg reflection, emission such as fluorescent x-rays 9 11 and electrons 12 from the crystal is strongly modulated, being maximum (minimum) when the antinodal (nodal) planes coincide with positions of the atoms in the crystal or on the surface. By measuring the angular dependence of the intensity of the emitted fluorescence and comparing with the computed angular dependence, the standing wave field has been used as a structural probe to determine the positions of the impurity atoms in crystals [9 11, 13], adsorbed atoms on surfaces [14], atoms at a layer/substrate interface 15 and to study thermal effects such as broadening of atomic position due to thermal vibration [16] and order-disorder transitions [17]. Various applications of the x-ray standing wave (XSW) technique to problems relating to single crystal surfaces and interfaces may be found in recent reviews 18, 19].

The standing wave phenomenon was also observed in multilayer mirrors 20 22] and Langmuir Blodgett multilayer films 23]. This standing wave field was also used in different ways for analyzing the local structure of multilayers [24,25], density evaluation of deposited films on multilayers [26] and selective extended x-ray absorption fine structure analysis [27].

For a periodic multilayer system x-ray reflectivity (XRR) is used to determine bilayer periodicity, interface roughness and the fractional thickness of the layers in a bilayer. Interface roughness characterization by x-ray standing waves has been attempted for a Ni/C multilayer system 25]. However, the extracted parameters were not optimized. Matsusita et al. 28 used XSW to determine the density of impurity atoms in a multilayer structure. Here we present a combined reflectivity and standing wave characterization of a periodic multilayer system to extract various structural parameters. As an example we use a 20 -period $\mathrm{Pt} / \mathrm{C}$ multilayer system. Comparing with experimental data we show that, 
structural parameters extracted from x-ray reflectivity analysis cannot explain the Pt fluorescence yield excited by $\mathrm{x}$-ray standing waves. Explanation of the Pt fluorescence yield, additionally requires the presence of an amount of dissolved $\mathrm{Pt}$ in the C-layers. XSW analysis provides the amount of dissolved $\mathrm{Pt}$ in $\mathrm{C}$ and the average composition $\mathrm{Pt}_{x} \mathrm{C}_{1-x}$ of the C-layers. Probing a small quantity of material dissolved from one layer into the other layer of a layer-pair in a multilayer system is very important for magnetic multilayers where alternating layers are magnetic and nonmagnetic materials. A small amount (even a few percent) of magnetic impurity (either from the magnetic layer or external) in the nonmagnetic layer can change magnetic coupling and magnetoresistance significantly [29], presumably because of changes in the topology of the Fermi surface of the resulting alloys.. The importance of the combined XSW and XRR analysis is elucidated.

\section{THEORY}

We give a brief theoretical background for the x-ray standing wave generation inside a multilayer system. We mainly follow the treatment given by Dev et al. [30] for the formation of standing waves and resonance enhancement of x-rays in layered materials using the recursion method of Parratt [31]. Then we obtain the field intensity for a periodic multilayer system and compute the angular variation of fluorescence yield from constituent elements in the multilayers. The fluorescence yield profile depends on the structural parameters of the multilayer. A consistent set of microstructural parameters of the multilayer is obtained from the combined analysis of reflectivity and fluorescence yield.

\section{A. Reflection from a multilayer system}

If all interfaces are parallel in a multilayer system Fig. 1, a plane electromagnetic wave of frequency $\omega$ in a medium $j$ at a position $\mathbf{r}$ can be written as

$$
E_{j}(\mathbf{r})=E_{j}(0) \exp \left[i\left(\omega t-\mathbf{k}_{j} \cdot \mathbf{r}\right)\right]
$$

where $E_{j}(0)$ is the field amplitude at the top of the $j$-th layer.

For all $j$, the components of the wave vector, $\mathbf{k}_{j}=$ $\mathbf{k}_{j}^{\prime}-i \mathbf{k}_{j}^{\prime \prime}$, are given by

$$
k_{j, x}=\frac{2 \pi}{\lambda} \cos \theta ; k_{j, z}=\frac{2 \pi}{\lambda}\left(\epsilon_{j}-\cos ^{2} \theta\right)^{1 / 2}
$$

where $\theta$ is the glancing angle of incidence, $\lambda$ is the wavelength of the incident $\mathrm{x}$-rays and the dielectric function $\epsilon_{j}$ is given by

$$
\epsilon_{j}=1-2 \delta_{j}-i 2 \beta_{j}
$$

where

$$
\begin{aligned}
& \delta=\frac{r_{e} \lambda^{2}}{2 \pi} N_{0} \frac{\rho_{m}}{M}\left(Z+f^{\prime}\right)=\left(\lambda^{2} / 2 \pi\right) r_{e} \rho \\
& \beta=\frac{r_{e} \lambda^{2}}{2 \pi} N_{0} \frac{\rho_{m}}{M} f^{\prime \prime}=(\lambda / 4 \pi) \mu
\end{aligned}
$$

In Eqn.(4) $\mathrm{N}_{0}$ is Avogadro's number, $\rho_{m}$ is the mass density of the element in the layer with atomic number $\mathrm{Z}$ and atomic weight M. $f^{\prime}$ and $f^{\prime \prime}$ are the real (dispersive) and the imaginary (absorption) anomalous dispersion factors, respectively. $\rho$ is the electron density (including dispersion) and $\mu$ is the linear absorption coefficient for the incident photons in the medium. $r_{e}$ is the classical electron radius. We consider the medium for the incident beam to be vacuum with $\epsilon_{0}=1$.

For the s-polarization of the electric field and smooth interfaces the complex coefficient of reflection $r_{j}$ and transmission $t_{j}$, being the ratio of electric fields at the $j, j+1$ interface, are given by Fresnel's formulas

$$
\begin{aligned}
& r_{j}=\frac{k_{j, z}-k_{j+1, z}}{k_{j, z}+k_{j+1, z}} \\
& t_{j}=\frac{2 k_{j, z}}{k_{j, z}+k_{j+1, z}}
\end{aligned}
$$

For small $\delta_{j}, \beta_{j}$ approximation, no distinction need be made between s-polarization and p-polarization [31].

For rough surfaces these expressions are to be modified. There are several methods for obtaining modified expressions. In a well-known method [32,33 $r_{j}$ is multiplied by a factor $S_{j}$ given by

$$
S_{j}=\exp \left[-2 \sigma_{j}^{2} k_{j, z} k_{j+1, z}\right]
$$

where $\sigma_{j}$ is the root-mean-square deviation of the interface atoms from the perfectly smooth condition. An expression like Eqn.(7) is only valid for small roughnesses $\left(\sigma_{j}\left|k_{j, z}\right|<1\right)$.

For the modification of $t_{j}$, it is to be multiplied by

$$
T_{j}=\exp \left[\sigma_{j}^{2}\left(k_{j, z}-k_{j+1, z}\right)^{2} / 2\right]
$$

So far we have discussed the reflection and refraction at a single interface. For a multilayer system, involving multiple interfaces, the electric fields at all the interfaces can be obtained from either a recursion relation or from a matrix formalism. In the following we will use the method of recursion relation. In the recursion method [31,34, the transmitted field $E_{j}^{t}$ and the reflected field $E_{j}^{r}$ at the top of the $j$-th layer are found from the relations :

$$
\begin{gathered}
E_{j}^{r}=a_{j}^{2} X_{j} E_{j}^{t}, \\
E_{j+1}^{t}=\frac{a_{j} E_{j}^{t} t_{j} T_{j}}{1+a_{j+1}^{2} X_{j+1} r_{j} S_{j}}
\end{gathered}
$$


and

$$
X_{j}=\frac{\left(r_{j} S_{j}+a_{j+1}^{2} X_{j+1}\right)}{1+a_{j+1}^{2} X_{j+1} r_{j} S_{j}}
$$

where

$$
a_{j}=\exp \left(-i k_{j, z} d_{j}\right)
$$

$d_{j}$ being the thickness of the $j$-th layer. For the substrate $E_{l}^{r}=X_{l}=0$.

The electric field amplitudes $E_{j}^{t}$ (transmitted) and $E_{j}^{r}$ (reflected) can be computed from the knowledge of $\lambda, \theta$, $\epsilon_{j}$ 's, the thickness of the layers $\left(d_{j}\right.$ 's) and the interface roughness ( $\sigma_{j}$ 's) using Eqn.(2) through Eqn.(12) and the reflectivity $R$ is then obtained from the ratio of E-fields outside the surface :

$$
R(\theta)=\left|E_{0}^{r} / E_{0}^{t}\right|^{2}
$$

For reflectivity from a periodic synthetic multilayer system involving interface roughness, this treatment is essentially equivalent to that of Underwood and Barbee [35.

For a periodic multilayer system, below the critical angle of incidence, $\theta_{1}^{c}=\sqrt{2 \delta_{1}}$, there exists an evanescent wave below the surface and total external reflection of the incident beam occurs $\left(\left|E_{o}^{r}\right| \approx\left|E_{o}^{t}\right|\right)$. The interference between $E_{o}^{r}$ and $E_{o}^{t}$ can form standing waves above the surface [22]. For $\theta>\theta_{1}^{c}$, the incident beam penetrates into the first layer of the multilayer system. When $\theta_{1}^{c} \geq \theta_{2}^{c}$, the incident beam penetrates into the multilayer system for $\theta>\theta_{1}^{c}$. If $\theta_{2}^{c} \geq \theta_{1}^{c}$, there is the possibility of resonance enhancement of x-rays in medium ' 1 ' for $\theta_{1}^{c}<\theta<\theta_{2}^{c}$ 30,34,36. For $\theta$ greater than both $\theta_{1}^{c}$ and $\theta_{2}^{c}$, the x-ray beam penetrates into the multilayer and if the multilayer is periodic, Bragg diffractions can occur [35].

For a periodic multilayer system of x-ray reflectors the multilayer period consists of one low and one high electron density alternating layers (say, $\mathrm{Pt} / \mathrm{C} / \mathrm{Pt} / \mathrm{C}$...), the higher density layer works as a marker and the low density layer works as a spacer. This arrangement makes the system an artificial periodic structure. Therefore, in the reflectivity from such a periodic multilayer system, Bragg peaks appear at positions determined by Bragg's law (including refraction and absorption).

$$
2\left(d_{1} k_{1, z}^{\prime}+d_{2} k_{2, z}^{\prime}\right)=2 n \pi
$$

or

$$
2\left(d_{1} \sin \theta_{1}+d_{2} \sin \theta_{2}\right)=n \lambda
$$

where the period of the multilayer is $d=d_{1}+d_{2}$ and $\mathrm{n}$ is the order of reflection.

It is well known from the dynamical theory of x-ray diffraction from perfect crystals that [8] a standing wave field is set up in the crystal during diffraction. The antinode position of this wave changes over half the unit-cell distance in passing the diffraction peak. This is also true for x-ray diffraction from a periodic multilayer system which will be illustrated later.

\section{B. Field Intensity}

The interference between the incident E-field $\left(E_{j}^{t}\right)$ and the reflected E-field $\left(E_{j}^{r}\right)$ can form standing waves within any layer. In order to obtain this standing wave field in the $j$-th layer one needs to know the fields $E_{j}^{t}$ and $E_{j}^{r}$ as a function of depth $(z)$. The total E-field at a point $\mathbf{r}$ in the $j$-th layer is given by

$$
E_{j}^{T}(\mathbf{r})=E_{j}^{t}(\mathbf{r})+E_{j}^{r}(\mathbf{r})
$$

where

$$
E_{j}^{t}(\mathbf{r})=E_{j}^{t}(0) \exp \left(-i k_{j, z} z\right) \exp \left[i\left(\omega t-k_{j, x} x\right)\right]
$$

and

$$
E_{j}^{r}(\mathbf{r})=E_{j}^{r}(0) \exp \left(+i k_{j, z} z\right) \exp \left[i\left(\omega t-k_{j, x} x\right)\right]
$$

Here the origin has been chosen to be on the interface at the top of the $j$-th layer. Thus $E_{j}^{t}(0)$ and $E_{j}^{r}(0)$ represent the transmitted and the reflected E-fields at the top of the $j$-th layer. $E_{j}^{t}(0)$ and $E_{j}^{r}(0)$ are readily obtained from the recursion relations (Eqn.(9) through Eqn.(12)). The field intensity $I(\theta, z)=\left|E_{j}^{T}(\mathbf{r})\right|^{2}$ is given by [30]

$$
\begin{aligned}
& I(\theta, z)=\left|E_{j}^{t}(0)\right|^{2}\left[\exp \left\{-2 k_{j, z}^{\prime \prime} z\right\}+\left|\frac{E_{j}^{r}(0)}{E_{j}^{t}(0)}\right|^{2} \exp \left\{2 k_{j, z}^{\prime \prime} z\right\}\right. \\
& \left.+2\left|\frac{E_{j}^{r}(0)}{E_{j}^{t}(0)}\right| \cos \left\{\nu(\theta)+2 k_{j, z}^{\prime} z\right\}\right],
\end{aligned}
$$

where $\nu(\theta)$ is defined by $\frac{E_{j}^{r}(0)}{E_{j}^{t}(0)}=\left|\frac{E_{j}^{r}(0)}{E_{j}^{t}(0)}\right| e^{i \nu(\theta)}$, i.e., $\nu(\theta)$ is the phase of the E-field ratio at the top of the $j$-th layer. If the absorption in the medium is ignored ( i.e, $k_{j, z}^{\prime \prime}=0$ ), Eqn (19) reduces to

$$
\begin{aligned}
& I(\theta, z)=\left|E_{j}^{t}(0)\right|^{2}\left[1+\left|\frac{E_{j}^{r}(0)}{E_{j}^{t}(0)}\right|^{2}\right. \\
& \left.+2\left|\frac{E_{j}^{r}(0)}{E_{j}^{t}(0)}\right| \cos \left\{\nu(\theta)+2 k_{j, z}^{\prime} z\right\}\right],
\end{aligned}
$$

It is clear from Eqn. (19) and (20) that a standing wave is generated within the $j$-th layer. The quantity within the square bracket in Eqn. (20) may attain a maximum value of 4 , for $\left|E_{j}^{r}(0) / E_{j}^{t}(0)\right|^{2}=1$. For small angles of incidence $(\theta)$, in some situations there are possibilities of resonance enhancement of $x$-ray intensity in the layer. This has been described in details by Dev et al. [30]. However, at $\theta \gg \theta_{1}^{c}$ and $\theta_{2}^{c},\left|E_{j}^{r}(0) / E_{j}^{t}(0)\right|^{2} \ll 1$ for a nonperiodic multilayer, and the field intensity is essentially given by 
the first term in Eq. (19) or (20) with a slight modulation from the second and the third terms. For such $\theta$ values the reflectivity is only significant when $\theta$ satisfies the Bragg condition for reflection from a periodic multilayer. Standing waves are set up in the multilayer when Bragg diffraction occurs. This can be seen from Eqn.(20) by inserting the Bragg condition [Eq. (14)]

$$
2\left(k_{1, z}^{\prime} d_{1}+k_{2, z}^{\prime} d_{2}\right)=2 k_{z}^{\prime} d=2 n \pi
$$

or

$$
k_{z}^{\prime}=\frac{n \pi}{d}
$$

where $k_{z}^{\prime}$ is the weighted average value for a layer-pair of the multilayer with periodicity $d=d_{1}+d_{2}$. While the magnitude of the E-field ratio varies to some extent for layer 1 and layer 2 of the bilayer, we can approximate this to be equal to its value just above the surface, [i.e., $\left|\frac{E_{j}^{r}(0)}{E_{j}^{t}(0)}\right|^{2} \approx\left|\frac{E_{0}^{r}}{E_{0}^{t}}\right|^{2}=R(\theta)$, from Eqn.(13)]. Now for normalized incident intensity, inserting the value of $k_{z}^{\prime}$ in Eqn.(20) we obtain (for $\mathrm{n}=1$ )

$$
I(\theta, z)=1+R(\theta)+2 \sqrt{R(\theta)} \cos \left\{\nu(\theta)+\frac{2 \pi}{d} z\right\}
$$

It is clear that Eqn.(22) now defines a standing wave within the multilayer with a periodicity $d$ and has the same form as that derived from the dynamical theory of x-ray diffraction from perfect crystals 9,18$]$. In the dynamical theory of x-ray diffraction the E-field in a medium is calculated by solving Maxwell's equations in that medium and obtaining solutions consistent with the Bragg's law. This E-field, then, describes the x-ray standing wave intensity as a function of angle over the region of the Bragg peak where the phase of $\frac{E^{r}}{E^{t}}(\theta), \nu(\theta)$, changes by $\pi$ radian [8, 9, 18, 37]. The actual value of $\nu(\theta)$ on the higher-angle side beyond the diffraction peak determines the position of the diffraction planes [37]. In order to show the similarity between the expressions for the standing wave intensity in the dynamical theory for perfect crystals and in the present case for multilayers we have inserted the Bragg's law into Eq. (20) and obtained Eq. (22), which is the well-known form obtained from the dynamical theory, where ' $1 / d$ ' is the magnitude of the reciprocal lattice vector for the concerned diffraction. The phase variation, $\nu(\theta)$, for the present case of multilayer is shown in Fig. 3. This has the similar form to that obtained from the dynamical theory 18, 37.

A periodic multilayer structure can be characterized by generating standing waves within the multilayer and measuring the standing-wave-excited fluorescence yield from one or more elements present in the multilayer. This is explained in the following sections. For the computation of standing wave field intensity, $I(\theta, z)$, we will use the more rigorous form of Eq.(19).

\section{Examples of calculation}

In this section we present the results of calculations of various quantities in sections II.A and II. B using an example - a periodic multilayer system consisting of 20 bilayers of $\mathrm{Pt} / \mathrm{C}$ on a glass substrate. The discussions presented here are general and not restricted to only $\mathrm{Pt} / \mathrm{C}$ multilayers.

For multilayers, earlier analyses were performed assuming the same roughness for both types of interfaces $(\mathrm{A} / \mathrm{B}$ and $\mathrm{B} / \mathrm{A})$ in the multilayer $(\mathrm{A} / \mathrm{B} / \mathrm{A} / \mathrm{B} \ldots)$ [34, 38]. In general, these values should be different. Surface free energy of the materials, $\sigma_{A}$ and $\sigma_{B}$, partly control the interface morphology during the growth. If $\sigma_{A}<\sigma_{B}$, it is the wetting condition for the growth of material A on material $\mathrm{B}$ and a nonwetting condition for the growth of material B on material A. Thus A-on-B (A/B) interface is expected to be smoother. The situation would be reverse for $\sigma_{A}>\sigma_{B}$. Indeed, high resolution electron microscopy on $\mathrm{W} / \mathrm{C}$ multilayers shows that the interface of $\mathrm{C}$ growing on $\mathrm{W}$ is much sharper than that of $\mathrm{W}$ growing on $\mathrm{C}$ [39]. It must be noted that $\sigma_{W}>\sigma_{C}$. However, other factors such as growth temperature and interdiffusion or chemical reaction between species across the interface also affect the interface roughness [40]. In any case, there is no reason to assume the interface roughness for both types of interfaces to be equal. Here we assume different roughnesses for the Pt-on-C $\left(\sigma_{1}\right)$ and the C-on$\mathrm{Pt}\left(\sigma_{2}\right)$ interfaces. It will be shown later that we indeed get a better fit to experimental data when $\sigma_{1}$ and $\sigma_{2}$ are allowed to be different.

In Fig. 2, we show the simulated reflectivity curve for smooth surface and interfaces alongwith those for several sets of values of surface and interface roughness. Total external reflection at low angles and multilayer Bragg peaks upto fourth order are seen. The higher order peaks are more drastically affected by the surface $\left(\sigma_{0}\right)$ and interface roughness $\left(\sigma_{1}, \sigma_{2}\right)$. The spacing between Bragg peaks is determined by the periodicity or the bilayer thickness $(d)$. So these parameters can be determined from the reflectivity data by a least-squares fitting procedure. In these computations we have used $\epsilon_{P t}=1-$ $\left(2.302 \times 10^{-5}\right)-\mathrm{i}\left(2.596 \times 10^{-6}\right)$ and $\epsilon_{C}=1-(3.016 \times$ $\left.10^{-6}\right)-\mathrm{i}\left(8.138 \times 10^{-10}\right), \quad\left(\rho_{P t}=5.05\right.$ electrons $/ \AA^{3}$, $\rho_{C}=0.698$ electrons $\left./ \AA^{3}\right), \lambda=0.709 \AA$ ( $\left(M o K_{\alpha_{1}} \mathrm{x}\right.$-rays) and $d=43 \AA\left(d_{1}=17 \AA, d_{2}=26 \AA\right)$. X-ray standing wave intensities are shown in Fig. 3 over the first Bragg peak region $\left(\theta=0.3^{\circ}\right.$ to $\left.\theta=0.6^{\circ}\right)$ at several angles shown on the reflectivity curve in the inset. The variation of phase, $\nu(\theta)$, of $\frac{E_{0}^{r}(0)}{E_{0}^{t}(0)}$ and $\frac{E_{1}^{r}(0)}{E_{1}^{t}(0)}$ are shown in the second inset of Fig. 3. The field intensity, $I(z)$, can be obtained using $R(\theta)$ and $\nu(\theta)$ from the insets and Eqn.(22). However, we have used the more rigorous Eqn.(19) to compute the field intensity $I(z)$ at several values of $\theta$. At an angle away from the strong reflection region (a) the field intensity, $I(z)$, has a weak modulation around a value of unity. At the low-angle side of the diffraction 
peak (b), there are antinodes of the standing wave field in the C-layers (nodes in the Pt layers). As $\theta$ increases $(\mathrm{b} \rightarrow \mathrm{c} \rightarrow \mathrm{d} \rightarrow \mathrm{e})$, the antinodes shift inward and finally coincide with the Pt-layers. The field intensity over the Pt-layers gradually increases as $\theta$ increases. The integrated field intensity in the Pt-layers,

$$
I_{P t}(\theta)=\sum_{j=o d d} \int_{0}^{d_{j}} I_{j}(\theta, z) d z
$$

is shown in Fig. 1. $I_{P t}(\theta)$ for smooth surfaces and interfaces $\left(\sigma_{0}=\sigma_{1}=\sigma_{2}=0\right)$ and for several sets of $\sigma_{0}, \sigma_{1}, \sigma_{2}$ values also shown. It is clearly seen that the field intensity $I(\theta)$ variation with $\theta$ is sensitive to surface and interface roughness. The integrated field intensity over the carbon layers,

$$
I_{C}(\theta)=\sum_{j=e v e n} \int_{0}^{d_{j}} I_{j}(\theta, z) d z
$$

for $\sigma_{0}=\sigma_{1}=\sigma_{2}=0$ is also shown in Fig. 田. It is noticed that the field intensity in the Pt-layers peaks at the high-angle edge while the intensity in the C-layers peaks at the low-angle edge of the reflectivity peak. This opposite trend holds the clue for the determination of the concentration of any dissolved Pt in C-layers.

Our objective is to find the $\mathrm{Pt}$ distribution in the $\mathrm{Pt} / \mathrm{C}$ multilayer. In the dipole approximation, fluorescence yield from an atom is proportional to the field intensity on the atom. Thus with the measurement of fluorescence yield from $\mathrm{Pt}$, it is possible to determine the $\mathrm{Pt}$ distribution. Fluorescence yield from $\mathrm{Pt}$ in the Pt layers should follow curve '1' in Fig. A, while fluorescence yield from $\mathrm{Pt}$ in the C-layers should follow curve '2'. So the effective shape of the fluorescence yield curve will depend on relative concentrations of $\mathrm{Pt}$ in the Pt-layers and the C-layers.

Interface roughness can be due to actual roughness or diffusion across the interface. The $\mathrm{Pt}$ distribution, $f(z)$, with interface roughnesses $\sigma_{1} \neq \sigma_{2}$ is schematically shown in Fig. 5. It is obvious that a fraction of $\mathrm{Pt}$ is in the C-layers near the interface. The fluorescence yield of $\mathrm{Pt}$ generated $\left(I^{f g}\right)$ from any depth is proportional to the product of the field intensity and $\mathrm{Pt}$ concentration at that depth.

$$
I_{j}^{f g}(\theta, z)=C I_{j}(\theta, z) f_{j}(z)
$$

where $C$ is a constant. The fluorescence yield detected outside the sample is given by

$$
I_{j}^{f d}(\theta, z)=C I_{j}(\theta, z) f_{j}(z) \times \exp \left[-\frac{\mu_{\text {out }}}{\sin \alpha}\left(\sum_{m=0}^{j-1} d_{m}+z\right)\right]
$$

with $d_{0}=0$ and the depth integrated detected fluorescence yield is

$$
\begin{aligned}
& I^{f d}(\theta)=C \sum_{j=1}^{N} \exp \left[-\frac{\mu_{\text {out }}}{\sin \alpha}\left(\sum_{m=0}^{j-1} d_{m}\right)\right] \times \\
& \int_{0}^{d_{j}} I_{j}(\theta, z) f_{j}(z) \exp \left(-\frac{\mu_{\text {out }}}{\sin \alpha} z\right) d z
\end{aligned}
$$

where $\alpha$ is the angle between the sample surface and the direction of the fluorescence detector from the center of the sample surface, and $\mu_{\text {out }}$ is the weighted average linear absorption coefficient for the outgoing (fluorescent) photons.

The distribution of $\mathrm{Pt}$ concentration over the bilayers across the Pt-on-C interface is given by

$$
f_{1}(z)=\frac{1}{2}\left[1-\operatorname{erf}\left(\frac{z}{\sqrt{2} \sigma_{1}}\right)\right]
$$

for $-d_{1} \leq z \leq d_{2}, z=0$ is on the Pt-on-C interface. $\sigma_{1}$ is the $\mathrm{Pt}$-on- $\mathrm{C}$ interface roughness. $\mathrm{Pt}$ distribution across the $\mathrm{C}$-on-Pt interface is given by

$$
f_{2}(z)=\frac{1}{2}\left[1+\operatorname{erf}\left(\frac{z}{\sqrt{2} \sigma_{2}}\right)\right]
$$

for $-d_{2} \leq z \leq d_{1}$ where $z=0$ is taken on the C-on-Pt interface. $\sigma_{2}$ is the C-on-Pt interface roughness. The total Pt distribution $f(z)$ over the bilayer and two interfaces is schematically shown in Fig. 5. $f(z)=f_{1}(z)+f_{2}(z)$ in the C-layers whereas in the Pt layer $f(z)=f_{1}(z)$ or $f_{2}(z)$, whichever is lower. The interface roughnesses $\sigma_{1}$ and $\sigma_{2}$ are those used in the analysis of reflectivity. Now that the Pt distribution, $f(z)$, over the total thickness of multilayer is defined, the integrated detected fluorescence yield, $I^{f d}(\theta)$, can be computed using Eqn. (27). The Pt fluorescence yield computed for this distribution of Pt over the first order Bragg reflection angular region is shown in Fig. 6 .

The solid curve $\left(\sigma_{0}=3, \sigma_{1}=5, \sigma_{2}=3 \AA\right)$ in Fig. 6 shows the computed fluorescence yield profile for $\mathrm{Pt}$ only in the Pt-layers. In this calculation the effect of roughness enters only in the computation of field intensity and the contribution to fluorescence yield from $\mathrm{Pt}$ in the $\mathrm{C}$ layer due to interface broadening is neglected. This means, in the Eqn.(27), only the sum over $j=$ odd layers have been considered. Sum over all layers contain the fluorescence yield contribution from $\mathrm{Pt}$ distributed in the C-layers as well. The fluorescence yield curve including this contribution is shown by the dashed line $\left(f_{c}=1\right.$, the significance of $f_{c}$ will be discussed later).

The possibility of a small amount of dissolved $\mathrm{Pt}$ in the C-layers, in addition to the $\mathrm{Pt}$ in the interface profile, has not yet been taken into account. In the computation of reflectivity the existence of such dissolved $\mathrm{Pt}$ in $\mathrm{C}$ should enter as a change in electron density of the C-layers. However, due to the low electron density of $\mathrm{C}\left(0.698\right.$ electrons $\left./ \AA^{3}\right)$, reflectivity is not very sensitive even to a relatively large change in C-layers electron density. Reflectivity for a $15 \%$ higher electron density 
(0.803 electrons $/ \AA^{3}$ ) of the C-layers, shown in Fig.7, is hardly distinguishable from that for pure $\mathrm{C}$ electron density. Moreover, the electron density of the C-layers not only depends on the amount of dissolved $\mathrm{Pt}$, but also on the change in C-layers thickness upon Pt incorporation. The electron density can also change due to incorporation of ambient atoms (e.g. Ar) during multilayer deposition [28]. Thus an accurate determination of the amount of $\mathrm{Pt}$ in the C-layers is difficult from the reflectivity measurement. However, with the x-ray standing wave method it is possible to determine the amount of dissolved $\mathrm{Pt}$ in the C-layers through the detection of its fluorescence. Here the detection of $\mathrm{Pt}$ is direct and the fluorescence yield variation with angle for $\mathrm{Pt}$ in the C-layers has the opposite trend compared to Pt in the Pt-layers (see Fig. (1). So an analysis of the shape of the measured Pt fluorescence yield curve can provide the amount of dissolved $\mathrm{Pt}$ in $\mathrm{C}$.

We assume the presence of some dissolved $\mathrm{Pt}$ in $\mathrm{C}$. Out of total $\mathrm{Pt}$ a fraction $f_{c}$ of $\mathrm{Pt}$ remains in the $\mathrm{Pt}$ layers and within the broadened interface regions of the C-layers, and the remaining fraction $\left(1-f_{c}\right)$ is dissolved uniformly in the C-layers. Pt fluorescence yields as a function of angle for $f_{c}=1,0.9$ and 0.8 are shown in Fig. 6. Later we will show with the experimental data that the fit to fluorescence yield improves when an $f_{c}<1$ is allowed in the least-squares fitting procedure. From the amount $\left(1-f_{c}\right)$ of $\mathrm{Pt}$ in the C-layers we can obtain the average composition $\left(\mathrm{Pt}_{x} \mathrm{C}_{1-x}\right)$ of the C-layers. In the present example, fractions $f_{c}=1,0.9$ and 0.8 correspond to $0,4.4$ and $8.7 \% \mathrm{Pt}$ in the C-layers, respectively. Keeping $f_{c}=1$, it is also possible to fit the fluorescence data assuming broader interfaces, i.e., allowing larger values of $\sigma_{1}$ and $\sigma_{2}$ in Eqns.(28) and (29). However, it would be inconsistent with the values of $\sigma_{1}$ and $\sigma_{2}$ obtained from the analysis of reflectivity data, as will be shown in section IV. In order to obtain a consistent set of microstructural parameters, it is necessary to allow, an $f_{c}<1 . f_{c}$ may be called coherent fraction and $\left(1-f_{c}\right)$ incoherent fraction in analogy with the XSW analysis with Bragg diffraction from single crystals 41.

\section{EXPERIMENTAL}

$\mathrm{Pt} / \mathrm{C}$ periodic multilayers with different bilayer period lengths $d$ ranging from $35 \AA$ to $47 \AA$ were made on float glass substrates, kept at room temperature, by dc magnetron sputtering specially designed for coating inner walls of cyllindrical surfaces. Two sputter sources of $\mathrm{Pt}$ and $\mathrm{C}$ are located at top and bottom of the cyllindrical vacuum chamber. Samples were grown at low argon pressure of 1 mbar. The deposition rate of $\mathrm{Pt}$ and $\mathrm{C}$ was $1 \AA /$ sec and $0.4 \AA /$ sec, respectively. The layer thickness during deposition was controlled using ion current and deposition time. Uniformity in the horizotal plane is achieved by rotating the sample while vertical uniformity is acieved by the mask. The overall thickness variation was found to be $<2 \%$ over an area of $10 \mathrm{~cm} \times 10 \mathrm{~cm}$. The control of the thickness of individual layers was within 1 $\AA$. A total of 20 layer pairs of $\mathrm{Pt} / \mathrm{C}$ were deposited in each case. The x-ray specular reflectivity measurements have been made on these samples [42] to determine the bilayer thickness and interface roughness. We have used one of these samples for the combined x-ray standing wave and reflectometry analysis.

Experiments were performed in our laboratory with a $18 \mathrm{~kW}$ Mo rotating anode x-ray source. The experimental set-up is schematically shown in Fig. 8. Monochromatic Mo $K_{\alpha_{1}}$ beam is obtained with the help of an asymmetrically cut $\mathrm{Si}(111)$ crystal monochromator. The asymmetrically-cut crystal reduces the divergence of the monochromatized beam and is in standard use in X-ray standing wave experiments [9]. The incident beam on the sample has an angular divergence of $0.006^{\circ}$. The vertical beam width is kept as small as $100 \mu m$. Reflected x-rays were detected with a $\mathrm{NaI}(\mathrm{Tl})$ detector and the $\mathrm{Pt} L_{\alpha}$ fluorescent x-rays were detected with a $\mathrm{Si}(\mathrm{Li})$ detector. The reflected $\mathrm{x}$-rays and the fluorescent $\mathrm{x}$-rays were collected simultaneously at each angle. Control of the instruments for the operation of the HUBER diffractometer and data collection is obtained through a PC using Turbo C programming for IEEE and RS-232 protocols. More details about the set-up has been presented elsewhere 433. The average exit angle $\alpha$ (the inclination of the $\mathrm{Si}(\mathrm{Li})$ detector with respect to the sample surface) for fluorescent photons was $50^{\circ}$.

\section{RESULTS AND DISCUSSIONS}

The experimental reflectivity data and the fitted theoretical reflectivity curve (Theory-1) are shown in Fig. 9 . Bragg peaks upto the third order are seen. The small oscillations are due to the total thickness of the multilayer. Experimental data have been fitted by allowing the variation in the electron density, layer thickness, and surface and interface roughnesses of the layers. From the least-squares fitting the values of the parameters have been extracted. This fitting gives the Pt-layers density $\rho_{1}=4.95$ electrons $/ \AA^{3}$, thickness $d_{1}=16.8 \AA$ and C-layers density (fixed) $\rho_{2}=0.698$ electron $/ \AA^{3}$, thickness $d_{2}=26.1$ $\AA$, $\sigma_{1}=4.5 \AA$ and $\sigma_{2}=2.9 \AA$. So the bilayer thickness is $42.9 \AA$. The third order peak position does not fit properly. This may be due to the multilayer having a slight variation in bilayer thickness along the growth direction.

It has been demonstrated that in case of single layer films the roughness is correlated with the thickness of the film [40,44]. But in the case of multilayer systems with alternating marker and spacer layers the roughness becomes complicated depending on the types of material, their diffusion properties, reaction and growth behavior [40]. It has been shown that in a W/C multilayer system the $\mathrm{W}$-on-C interface is more rough than the $\mathrm{C}$ - 
on-W interface [39]. Fundamentally this is expected because of the nonwetting condition in the surface free energy $\left(\sigma_{W}>\sigma_{C}\right)$ for the growth of $\mathrm{W}$ on $\mathrm{C}$. In our case $\sigma_{P t}>\sigma_{C}$ and we also observed the same trend: the Pt-on-C interface is more rough $\left(\sigma_{1}=4.5 \AA\right)$ than the Con-Pt interface $\left(\sigma_{2}=2.9 \AA\right)$. Pt electron density for this sample is 4.95 electron $/ \AA^{3}$, which is lower than that of pure Pt electron density of 5.05 electron $/ \AA^{3}\left(\rho_{m}=21.5\right.$ $\mathrm{gm} / \mathrm{cc}$ ). In general, thin films tend to have a lower density compared to pure bulk material. Additionally, interdiffusion across the interfaces leading to a mixed layer would decrease the Pt-layers density and increase the Clayers density.

The $\mathrm{Pt} L_{\alpha}$ fluorescence yield has been measured over an angular region containing the first order Bragg peak and analyzed as follows. From the spectrum at each angle in the multichannel analyzer only $\mathrm{Pt} L_{\alpha}$ peak is selected. These peaks at all angles are fitted and the backgroundsubtracted area is determined. This area gives the yield. This raw yield data have been corrected for footprint, probing thickness variation and finite detector aperture. These corrections are explained at the end of this section. This corrected $\mathrm{Pt} L_{\alpha}$ fluorescence yield vs. angle along with reflectivity over first Bragg peak is shown in Fig. 10. We fit the fluorescence yield data based on the model described earlier. This model incorporates all the parameters extracted from the reflectivity fit. That means that the density, thickness, surface and interface roughness etc.. of the layers are kept intact. Here we have considered the contribution of roughness as error functions [Eqns. (28) and (29)] at both the interfaces with $\sigma_{1}=4.5$ $\AA$ and $\sigma_{2}=2.9 \AA$. These $\sigma$-values are the roughness values obtained from the analysis of reflectivity. (It is well known that reflectivity calculations using explicit errorfunction concentration profile at the interface and the flat interface reflection coefficient multiplied by a DebyeWaller function [Eqn. (7)] are equivalent [45]). If we consider that there is no dissolved $\mathrm{Pt}$ in the C-layers (i.e. $f_{c}=1$ ), we do not obtain a good fit. The best fit is obtained with the model with a uniform mixing of $\mathrm{Pt}$ in the C-layers with $f_{c}=0.87$. This means that $13 \%$ of total $\mathrm{Pt}$ is dissolved within the C-layers. Converted to atomic concentration this corresponds to the average composition $\mathrm{Pt}_{0.05} \mathrm{C}_{0.95}$ of the carbon layer. It should be noted that $\mathrm{Pt}$ concentration in the C-layers is actually higher near the interface. This concentration varies with the distance from the interface and can be easily determined from the distributions [Eqns.(28) and (29)].

In order to show the sensitivity of the fluorescence yield curve to the $\mathrm{Pt}$ concentration in the C-layers, we also show the plots for $\mathrm{Pt}_{0.03} \mathrm{C}_{0.97}$ and $\mathrm{Pt}_{0.07} \mathrm{C}_{0.93}$ in Fig. 10 . They are distinctly different from the data and the fitted curve for $\mathrm{Pt}_{0.05} \mathrm{C}_{0.95}$. This clearly shows that the uncertainty in the estimated $\mathrm{Pt}$ concentration of $5 \%$ is smaller than $2 \%$. In the fitting of data the weighted Rfactors are $0.041,0.031,0.023,0.024$ and 0.029 for 3,4 , 5, 6 and $7 \% \mathrm{Pt}$, respectively. It is noticed from Fig. 10 that with increasing $\mathrm{Pt}$ concentration in $\mathrm{C}$, Pt fluores- cence yield increases on the low-angle edge and decreases on the high-angle edge of the reflectivity curve. This can be easily understood from Fig. 3. At angular position 'b' on the reflectivity curve, X-ray intensity is high in the C-layers and low in the Pt-layers. However, if there is no Pt in the C-layers, there would be no Pt fluorescence emission from there. As some $\mathrm{Pt}$ migrates from the Pt-layers to C-layers, the amount of $\mathrm{Pt}$ present in the C-layers would produce strong fluorescence emission. That is why increasing Pt concentration in the C-layers produces higher fluorescence yield at this angular position 'b' as seen in Fig. 10. It is also noticed from Fig. 3 that the maximum field intensity in the C-layers is much higher than the maximum field intensity in the Pt-layers (see also Fig. (1). This is due to lower absorption of Xrays in the C-layers. Due to this fact, a given amount of $\mathrm{Pt}$ in the C-layers produces a stronger fluorescence signal than the same amount in the Pt-layers when the X-ray intensities are maximum in the respective layers.

Probing the quantity of material dissolved from one layer into the other layer of a layer-pair in a multilayer system is not only important for optical mirrors and devices, but also very crucial for magnetic multilayers where interface broadening and alloying within the layers affect magnetic properties of multilayers. In magnetic multilayers with alternating layers of magnetic and non-magnetic materials, a small amount (even a few percent) of magnetic impurity in the nonmagnetic layers can change the magnetic coupling and magnetoresistance. In fact, in magnetic multilayers with a wide range of $\mathrm{Cu}_{1-x} \mathrm{Ni}_{x}$ $(x=0.04$ to 0.42$)$ alloy spacer, the smallest amount of impurity $(x=0.04)$ has shown the largest change in magnetoresistance [29]. The magnetic impurity in the nonmagnetic layer of the multilayer may be an element other than the magnetic element present in the multilayer. Since $\mathrm{x}$ ray fluorescence can identify the element, the distribution of such impurity elements in the multilayer can be determined by XSW experiments [28].

It must be mentioned here that the fluorescence data can also be fitted, without assuming the dissolved fraction (i.e. keeping $f_{c}=1$ ), by allowing $\sigma_{1}$ and $\sigma_{2}$ to vary for the fluorescence fit. This fit is also shown in Fig. 10. However, the $\sigma$-values obtained from this fit ( $\sigma_{1}=8.9 A$, $\left.\sigma_{2}=4.2 \AA\right)$ are inconsistent with those obtained from the reflectivity fit. The computed reflectivity for these $\sigma$-values, as shown in Fig. 9 (Theory-2), is very different from the measured reflectivity. This shows that this set of larger $\sigma$-values does not represent correct interface roughness. This is probably the reason why a very large $\sigma$-value $(10 \AA)$ fitted the fluorescence data of Kawamura et al. 24]. Our results underline the necessity for the combined x-ray standing wave and reflectivity analysis of periodic multilayers. We suggest that a combined use of reflectivity and x-ray standing waves can provide the microstructural details of a periodic multilayer. The procedure to follow is: (i) obtain bilayer periodicity, fractional thickness of the high- $Z$ layer and surface and interface roughnesses from the reflectivity fit, (ii) interface 
roughness should not be constrained to be equal for both types of interfaces, and (iii) use the parameters obtained from the reflectivity fit and proceed for the the fluorescence data fit with the assumption of a dissolved fraction of one material in the other, either in uniform distribution or with any other improved distribution model. For a more accurate determination of this distribution, higher order Fourier components of the distribution can be determined by XSW measurements with higher order Bragg diffractions.

In order to fit the reflectivity data to Eqn.(13) and fluorescence data to Eqn.(27), the following corrections to data were applied: (i) Footprint correction 46] was applied to both reflectivity and fluorescence data. At very small angles the beam projection is larger than the sample area. So, only a fraction of incident photons are actually incident on the sample. After this correction, the data represent what they should be if all the photons were incident on the sample. (ii) The fluorescence data come from a relatively thin layer (thickness of the multilayer) compared to the beam penetration depth. Thus with the variation of $\theta$ the effective probe depth changes. To correct for that, fluorescence data are to be multiplied by $\sin \theta$ at each point. (iii) The fluorescence detector has a finite aperture and the fluorescent photons may come from a much larger sample area. The detector offers a varying effective solid angle for fluorescent photons originating from different parts of the sample surface. As the exposed sample area varies with $\theta$, this requires a correction which depends on detector aperture, detector distance from the sample and the sample length. In our case, over the $\theta$ range $\left(0.45^{\circ}-0.6^{\circ}\right)$ of the first order Bragg peak region this introduces only a minor correction for $1 \%$ variation in detected intensity.

\section{CONCLUSIONS}

For a periodic multilayer system with alternating layers of a high-Z and a low-Z element, Bragg diffraction of $\mathrm{x}$-rays occurs when the Bragg condition for the bilayer periodicity is satisfied. As in diffraction from a large perfect crystal, standing waves are set up in the multilayer while diffraction occurs. The antinodal (or nodal) planes of the standing wave are parallel to the layerplanes and have the periodicity equal to the multilayer period. On the low-angle side of the Bragg-reflection peak, the antinodal planes are within the layers with low$\mathrm{Z}$ element. As the angle of incidence advances through the diffraction peak the antinodal planes shift inward and finally coincide with the nearest layer of high-Z element of the layer-pairs. Emission processes, such as photoemission or fluorescence from atoms in the multilayer are modulated, over an angular region containing the Bragg peak, following the shift of the antinodal planes. Analysis of this modulation in the emission yield provides structural information about the multilayer. The usefulness of the combined application of x-ray reflectivity and x-ray standing wave techniques for the analysis of multilayer microstructures has been explained. Deficiencies of each technique can be overcome by the combined application of these techniques. XRR depends on the electron density difference between the layers of the bilayer. Where electron density of one layer of the layer-pair is very small compared to the other, reflectivity is not very sensitive to even a large fractional change of this electron density. Moreover the change of electron density is not necessarily due to the diffusion of atoms from the other layer of the layer-pair, it could also be due to other impurities incorporated during multilayer fabrication. Thus accurate determination of the layer composition from XRR technique is practically impossible. These aspects have been elucidated with an example of a 20 period $\mathrm{Pt} / \mathrm{C}$ multilayer. In the XSW technique, elements are directly identified. Thus the amount of dissolved $\mathrm{Pt}$ or any other impurity in the C-layers, such as Ar, often incorporated during multilayer fabrication, can be determined. As interface roughness drastically affects the higher order Bragg peaks and overall intensity at higher angles, interface roughnesses are more accurately determined by fitting the reflectivity data over a large range of angle of incidence. On the other hand, in the XSW analysis, if the amount of $\mathrm{Pt}$ in the C-layers is assumed to be solely within the broadened interface and treated as roughness, one obtains too large roughness values compared to those obtained from the reflctivity fit. Fixing the interface roughness values at those obtained from the $\mathrm{XRR}$ analysis and assuming the remaining $\mathrm{Pt}$ to be in uniform distribution in the $\mathrm{C}$-layers, the $\mathrm{Pt}$ concentration in the C-layers is determined. (More details about the elemental distribution, such as higher order Fourier components, can be obtained by XSW measurements with higher order Bragg peaks). Thus a combined analysis by XSW and XRR techniques removes the deficiencies of the individual techniques. For a 20 period $\mathrm{Pt} / \mathrm{C}$ multilayer system interface roughnesses ( Pt-on-C: $4.5 \stackrel{\circ}{A}$, C-on-Pt : $2.9 \stackrel{\circ}{A})$ and the C-layers composition $\left(\mathrm{Pt}_{0.05} \mathrm{C}_{0.95}\right)$ have been determined. Determination of a small quantity of impurity, even a few percent, in the spacer layer is particularly important in the magnetic multilayers.

\section{ACKNOWLEDGMENTS}

We thank Dr. G. Lodha and Prof. K. Yamashita for providing the $\mathrm{Pt} / \mathrm{C}$ multilayer sample.

* E-mail: bhupen@iopb.res.in; Fax: +91 - 674- 300142.

[1] E. Spiller, Appl. Phys. Lett. 20, 365 (1972). 
[2] T. W. Barbee, Synthetic Modulated Structure Materials (Academic, New York, 1985), p. 313.

[3] D. B. McWhan, Synthetic Modulated Structure, (Eds.) L. L. Chang and B. C. Giesser, (Academic, New York, 1985), Chap. 2. p. 43.

[4] M. B. Stearns, J. Appl. Phys., 55, 1729 (1984); see also the Proceedings of the International Conference on Magnetism, 1985, San Francisco (North-Holland, Amsterdam, 1985).

[5] C. M. Falco and I. K. Schuller, Synthetic Modulated Structure Materials (Academic, New York, 1985 ), and reference therein.

[6] Home page of Center for X-ray Optics, http://wwwcxro.lbl.gov

[7] M. von Laue, Roentgenstrahl-Interferenzen (Akademische Verlagsgesellschaft, Frankfurt, 1960).

[8] B. W. Batterman and H. Cole, Rev. Mod. Phys. 36, 681 (1964).

[9] B. W. Batterman, Phys. Rev. 133, A759 (1964).

[10] J. A. Golovochenko, B. W. Batterman and W. L. Brown, Phys. Rev. B10, 4239 (1974)

[11] S. K. Andersen, J. A. Golovochenko and M. F. Robbins, Phys. Rev. Lett. 37, 1141 (1976).

[12] M. J. Bedzyk, G. Materlik and M. V. Kovalchuk, Phys. Rev. B30, 2453 (1984).

[13] Th. Gog, T. Harasimowicz, B. N. Dev and G. Materlik, Europhys. Lett. 25, 253 (1994).

[14] P. L. Cowan, J. A. Golovochenko and M. F. Robbins, Phys. Rev. Lett. 44, 1680 (1980).

[15] E. Vlieg, A. E. M. J. Fischer, J. F. van der Veen, B. N. Dev and G. Materlik, Surf. Sci., 178, 36 (1986).

[16] M. J. Bedzyk and G. Materlik, Phys. Rev., B31, 4110 (1985).

[17] B. N. Dev, F. Grey, R. L. Johnson and G. Materlik, Europhys. Lett. 6, 311 (1988); B. N. Dev, Phys. Rev. Lett., 64, 1182 (1990).

[18] J. Zegenhagen, Surf. Sci. Rep., 18, 199 (1993).

[19] B. N. Dev, in X-ray and Inner-Shell Processes, (Eds.), R. L. Johnson, H. Schmidt-Boecking and B. F. Sonntag, AIP Conference Proceedings, 389, (1997) pp. 249-265.

[20] T. W. Barbee and W. K. Warburton, Mater. Lett. 3, 17 (1984).

[21] B. Lai, G. M. Wells, R. Readaelli, F. Cerrina, K. Tan, J. H. Underwood and J. Kortright, Nucl. Instrum. Methods, A266, 684 (1988).

[22] M. J. Bedzyk, D. H. Bilderback, G. M. Bommarito, M. Caffrey and J. S. Schildkraut, Science, 241, 1788 (1988).

[23] A. Iida, T. Matsushita and T. Isikawa, Jpn. J. Appl. Phys. 24, L675 (1985).

[24] J. B. Kortright and A. Fischer-Colbrie, J. Appl. Phys. 61, 1130 (1987).

[25] T. Kawamura and H. Takenaka, J. Appl. Phys. 75, 3860 (1994).

[26] S. I. Zheludeva, M. V. Kovalchuk, N. N. Novikova, and I. V. Bashelhanov, Rev. Sci. Instrum. 63, 1519 (1992).

[27] S. M. Heald and J. M. Tranquada J. Appl. Phys. 65, 290 (1989).

[28] T. Matsushita, A. Iida, T. Ishikawa. T. Nakagiri and K. Sakai, Nucl. Instrum. and Methods A246, 751 (1986).

[29] S. S. P. Parkin, C. Chappert and F. Herman, Mater. Res.
Soc. Symp. Proc., Vol 313 (1993) 179, and other publications in the same issue.

[30] B. N. Dev, A. K. Das, S. Dev, D. W. Schubert, M. Stamm and G. Materlik, Phys. Rev. B61, 8462 (2000).

[31] L. G. Parratt, Phys. Rev. 95, 359 (1954).

[32] B. Vidal and P. Vincent, Appl. Opt. 23, 1794 (1984).

[33] B. Pardo, T. Megademini and J. M. Andre, Rev. Phys. Appl. 23, 1579 (1988).

[34] D. K. G. De Boer, Phys. Rev. B44, 498 (1991).

[35] J. H. Underwood and T. W. Barbee, Jr. Appl. Opt., 27, 3027 (1981)

[36] Jin Wang, Michael J. Bedzyk and Martin Caffrey, Science, 28, 775 (1992).

[37] B. N. Dev and G. Materlik, in Resonance Anomalous Xray scattering: Theory and applications, Elsevier Science B. V., 119 (1994), (Eds) G. Materlik, C. J. Sparks and K. Fischer.

[38] A. Kroel, C. J. Sher, and Y. H. Kao, Phys. Rev. B33, 8579 (1988).

[39] Amanda K. Pettford-Long, Mary Beth Stearns, C. H. Chang, S. R. Nutt, D. G. Stearns, N. M. Ceglio and A. M. Hawryluk, J. Appl. Phys. 61,1422 (1987).

[40] D. E. Savage, N. Schimke, Y. H. Phang and M. G. Lagally, J. Appl. Phys. 71, 3283 (1992).

[41] According to the convention of x-ray standing wave analysis with Bragg diffraction from single crystals even the $\mathrm{Pt}$ in the C-layers at the broadened interface would be considered in the incoherent fraction.

[42] G. Lodha, A. Paul, S. Vitta, A. Gupta, R. Nandedkar, K. Yamashita, H. Kunieda, Y. Tawara, K. Tamura, K. Haga and T. Okajima, Jpn. J. Appl. Phys., 38, 289 (1999).

[43] P. V. Satyam, D. Bahr, S. K. Ghose, G. Kuri, B. Sundaravel, B. Rout and B. N. Dev, Current Sci., 69, 526 (1995); P. V. Satyam, Ph. D. Thesis (Utkal university, 1996).

[44] S. K. Sinha, Physica, A224, 140 (1996).

[45] P. Boher, P. Hindy and C. Schiller, J. Appl. Phys., 68, 6133 (1990).

[46] A. Gibaud, G. Vignaud and S. K. Sinha, Physica A49, 642 (1993). 


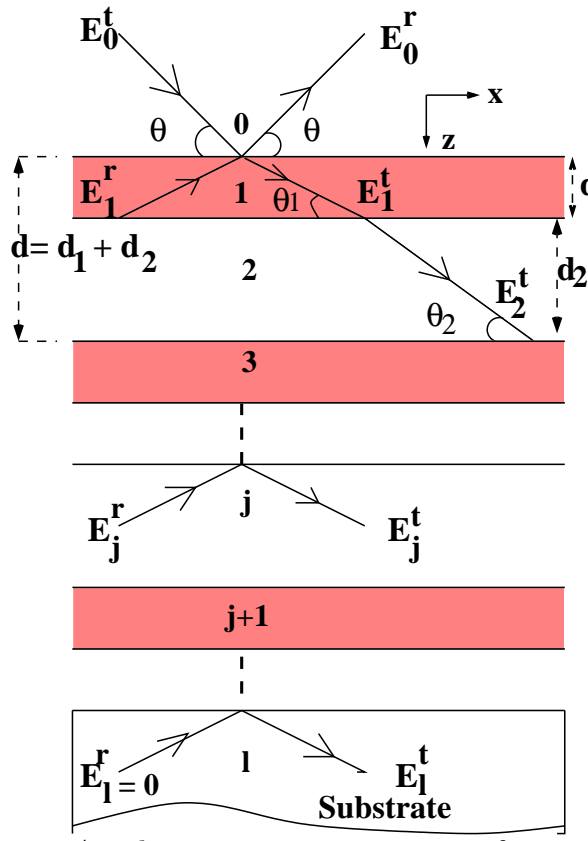

FIG. 1. A schematic representation of x-ray reflection from a multilayer system. See text for details.

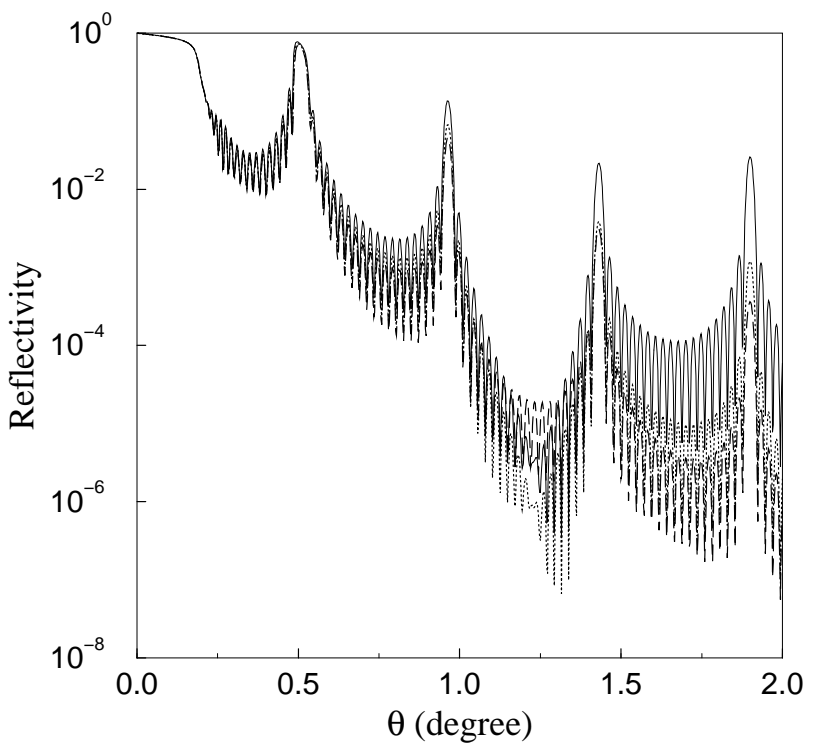

FIG. 2. Reflectivity from a 20 period Pt/C multilayer system with periodicity $d(43 \AA)=d_{1}(17 \AA)+d_{2}(26 \AA)$ and with surface and interface roughnesses $\left(\stackrel{\circ}{)} \sigma_{0}, \sigma_{1}, \sigma_{2} 0,0,03,3\right.$, $3(\ldots \ldots)$ and $3,5,30,0,0$ ( $3,3,3(\ldots \ldots)$ and 3,5 , $3(---)$.

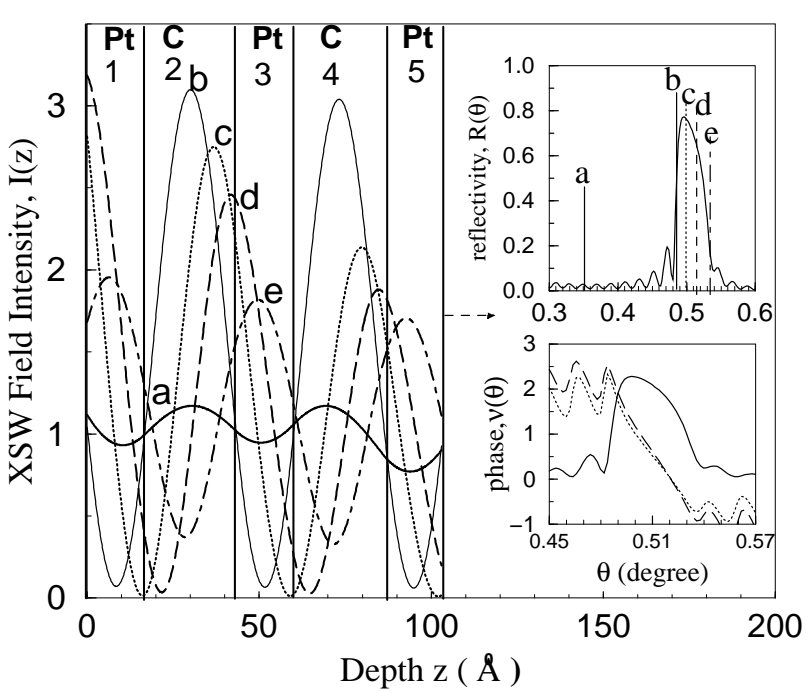

FIG. 3. X-ray standing wave field intensity distribution within the $\mathrm{Pt} / \mathrm{C}$ multilayer system, at different angles of incidence $\theta$ over the 1st order Bragg peak region (shown in the inset). (a) $\theta=0.35^{\circ}$ (—), (b) $\theta=0.486^{\circ}$ (—), (c) $\theta=0.500^{\circ}$ (.......), (d) $\theta=0.516^{\circ}(---)$, (e) $\theta=0.535^{\circ}$ (- -). The phases, $\nu(\theta)$, of E-field ratios $\frac{E_{0}^{r}}{E_{0}^{t}}(\ldots \ldots \ldots)$ and $\frac{E_{1}^{r}}{E_{1}^{t}}$ $(---)$ are also shown in the second inset, which also shows reflectivity $(\longrightarrow, \times 3)$. At a given depth $z$, the variation in field intensity with angle over the strong reflection region occurs mainly because of large variation in phase, $\nu(\theta)$. [ see Eqn.(22)].

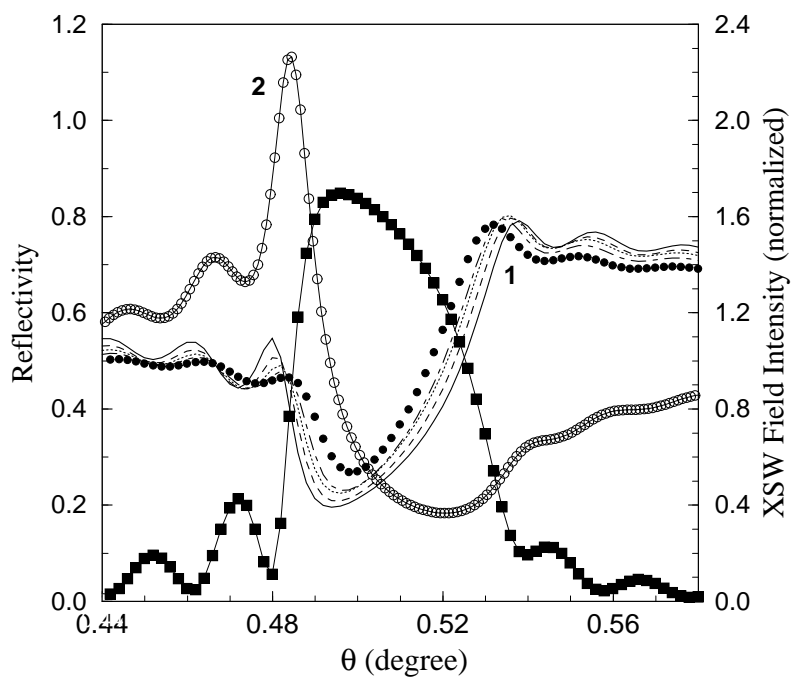

FIG. 4. Integrated XSW field intensity over the Pt-layers and over the C-layers for different surface and interface roughnesses for the $\mathrm{Pt} / \mathrm{C}$ multilayer system. Reflectivity over the 1st order Bragg peak (solid squares) for $\sigma_{0}=0, \sigma_{1}=0, \sigma_{2}=0$ $(A)$, integrated field intensity over Pt-layers with $\sigma_{0}, \sigma_{1}, \sigma_{2}$

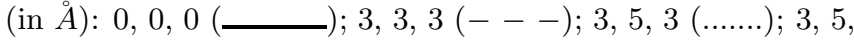
$5(---) ; 3,7,7(\bullet \bullet \bullet)$ and integrated field intensity over C-layers (connected open circles) for $\sigma_{0}=3, \sigma_{1}=5, \sigma_{2}=3(\stackrel{\circ}{A})$. 


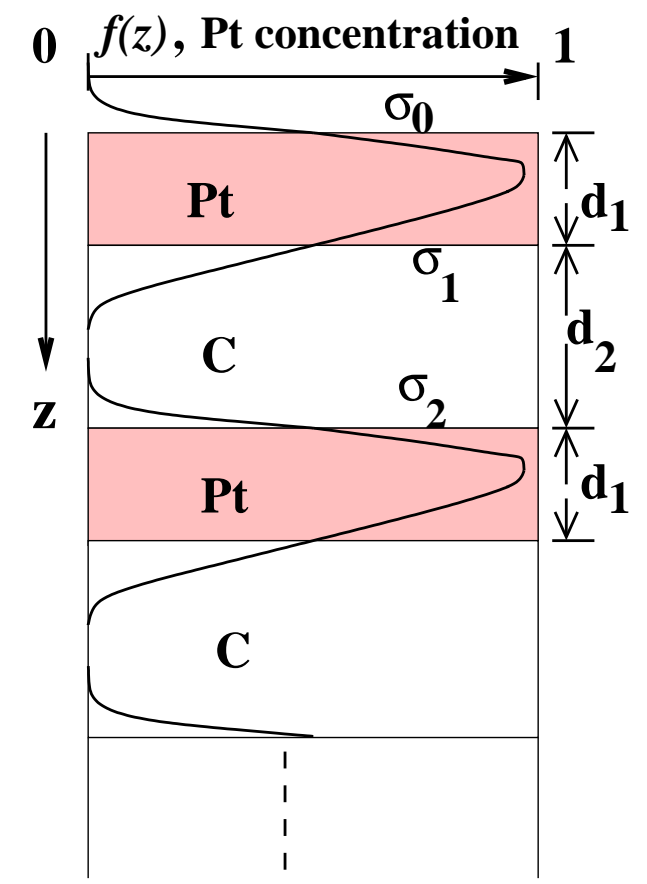

FIG. 5. Schematic diagram of Pt distribution, $f(z)$, with interface roughness over the bilayer period. $\sigma_{0}, \sigma_{1}$ and $\sigma_{2}$ are surface roughness, $\mathrm{Pt}$-on- $\mathrm{C}$ and $\mathrm{C}$-on-Pt interface roughnesses, respectively

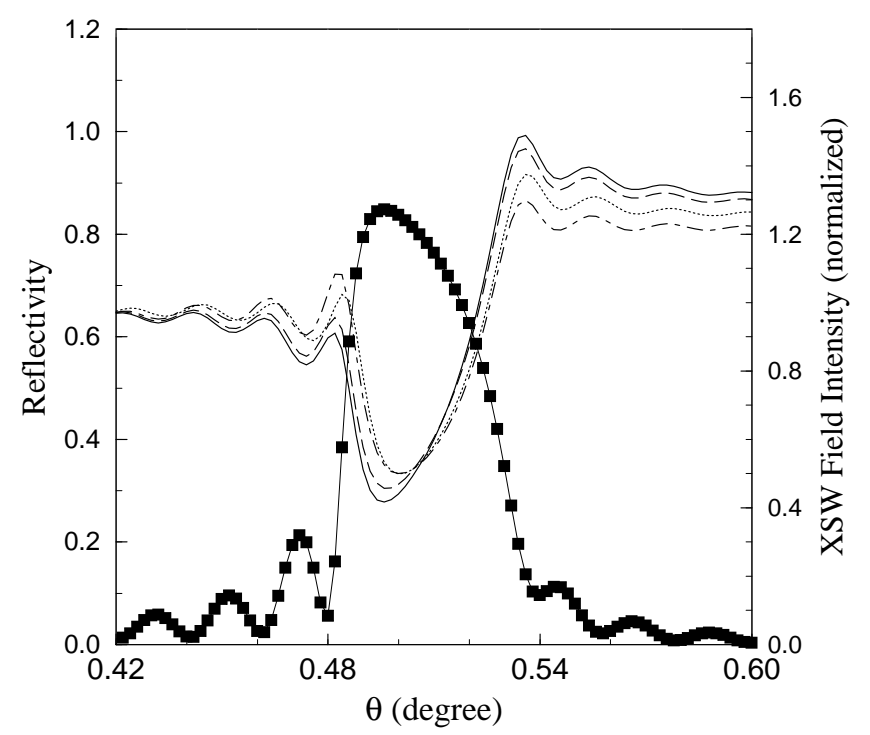

FIG. 6. Theoretical plots for Pt fluorescence yield, computed for the distribution of $\mathrm{Pt}$ in Fig. B, over the first order Bragg reflection angular region. Reflectivity (solid squares), Pt fluorescence yield integrated over Pt-layers with surface and interface roughnesses $\sigma_{0}=3 \stackrel{\AA}{A}, \sigma_{1}=5 \stackrel{\AA}{A}, \sigma_{2}=3 \AA$ ( Pt fluorescence yield integrated over the whole multilayer $\left(\sigma_{0}=3 \AA, \sigma_{1}=5 \AA, \sigma_{2}=3 \AA\right.$ and for $f_{c}=1(---), f_{c}=0.9$ $(\ldots \ldots)$ and $f_{c}=0.8(---)$. See text for details.

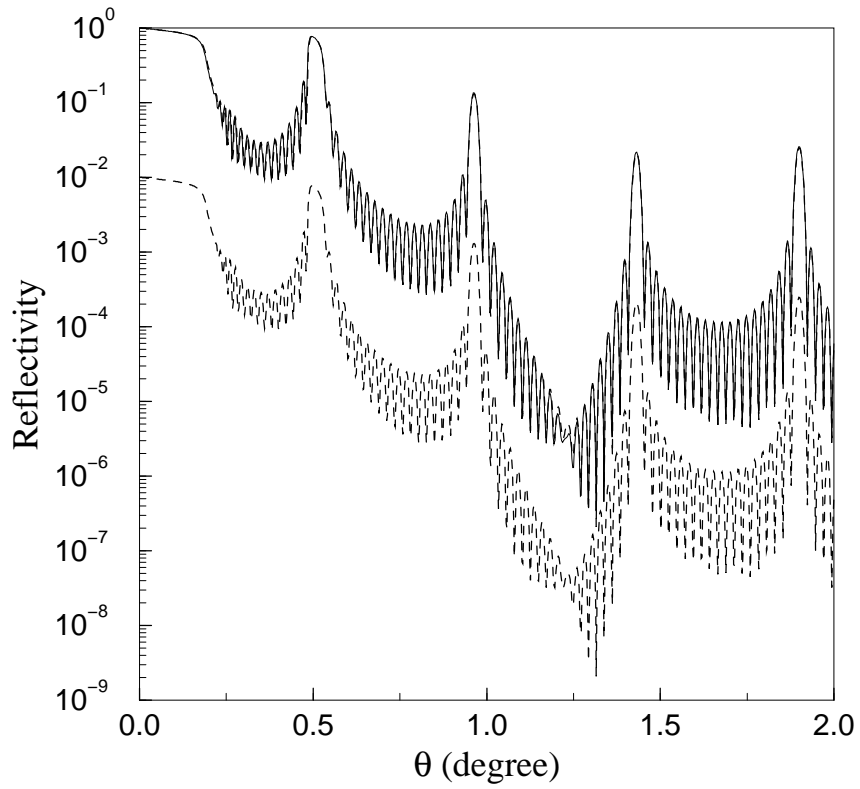

FIG. 7. Theoretical plots of reflectivity for different electron densities $\rho_{C}$ of the C-layers. $\rho_{C}=0.698$ electrons $/ \AA^{3}$ (—), $\rho_{C}=0.803$ electrons $/ \AA^{3}$ ( $15 \%$ higher compared to the actual density) $(---)$. Curves are vertically shifted by two orders. However, they are also shown in overlaping mode to demonstrate that they are practically indistinguishable.

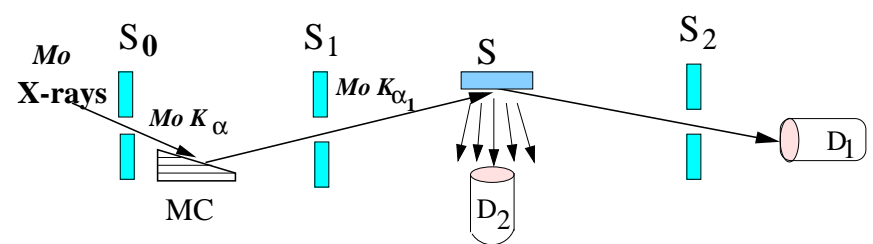

FIG. 8. A schematic view of the experimental set up with an asymmetric $\mathrm{Si}(111)$ crystal monochromator (MC) and incident x-rays from an $18 \mathrm{~kW}$ rotating Mo anode x-ray generator. Slits: $S_{0}, S_{1}$ (horizontal width $=4 \mathrm{~mm}$, vertical width $=100 \mu \mathrm{m}$ ), $S_{2}$ (horizontal width $=10 \mathrm{~mm}$, vertical width $=150 \mu \mathrm{m}) ; \mathrm{D} 1: \mathrm{NaI}(\mathrm{Tl})$ scintillation detector ; D2 : $\mathrm{Si}(\mathrm{Li})$ energy dispersive detector; S: sample. 


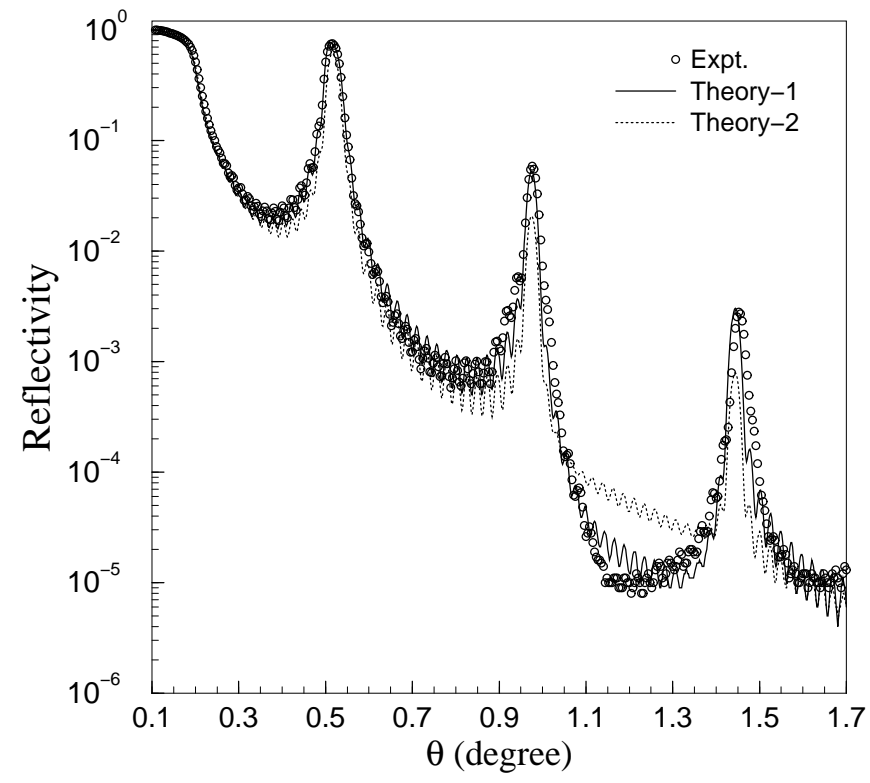

FIG. 9. Experimental reflectivity data ( $\circ \circ \circ)$ and fitted theoretical reflectivity curve (—) for a $\mathrm{Pt} / \mathrm{C}$ multilayer on a glass substrate with 20 bilayers. Parameters obtained from the fit: bilayer thickness $d=42.9 \AA$, Pt layer thickness $d_{1}=16.8 \AA$ and C-layers thickness $d_{2}=26.1 \AA$, surface roughness $\sigma_{0}=3 \AA$, Pt-on-C interface roughness $\sigma_{1}=4.5 \AA$ and C-on-Pt interface roughness $\sigma_{2}=2.9 \AA$. Theoretical reflectivity curve $(\ldots \ldots \ldots$.$) for \sigma_{0}=3 \AA, \sigma_{1}=8.9 \AA$ and $\sigma_{2}=4.2 \AA$ and all other parameters unchanged. See text for details.

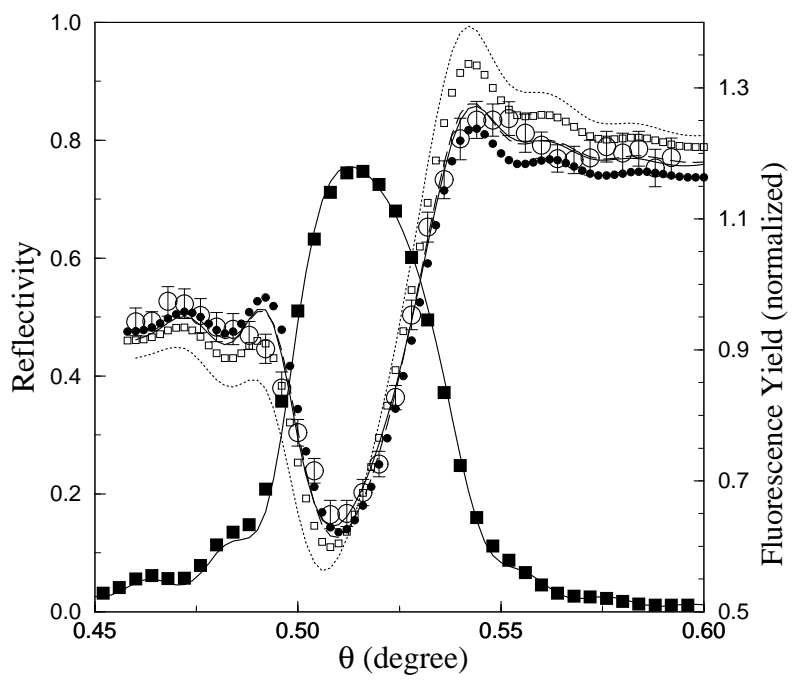

FIG. 10. Experimental Pt $L_{\alpha}$ fluorescence yield ( O O O ) and reflectivity (solid squares) vs angle of incidence $\theta$ over the first order Bragg reflection and the theoretical curves:

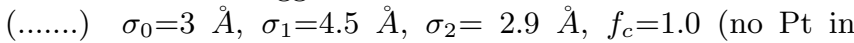
C-layers); (—) $\sigma_{0}=3 \stackrel{\circ}{A}, \sigma_{1}=4.5 \stackrel{\circ}{A}, \sigma_{2}=2.9 \AA \stackrel{\AA}{A} f_{c}=0.87$ $\left(\mathrm{Pt}_{0.05} \mathrm{C}_{0.95}\right) ;(---) \sigma_{o}=3 \stackrel{\AA}{\AA}, \sigma_{1}=8.9 \AA{ }^{\circ}, \sigma_{2}=4.2 \AA, f_{c}=1.0$. Also shown, (for $\sigma_{o}=3 \AA$, $\sigma_{1}=4.5 \AA$ and $\sigma_{2}=2.9 \AA$ ) are the curves (open squares) for $\mathrm{Pt}_{0.03} \mathrm{C}_{0.97}\left(f_{c}=0.935\right)$ and (filled circles) for $\mathrm{Pt}_{0.07} \mathrm{C}_{0.93}\left(f_{c}=0.844\right)$. Fluorescence curves have been normalized at $\theta=0.4^{\circ}$ as in Fig. 6. See text for details. 